\title{
Mitigação das emissões de amônia por zeólitas naturais durante a compostagem de dejetos de suínos
}

\author{
Diego Antonio Giacomini(1), Celso Aita(1), Stefen Barbosa Pujol(1), Sandro José Giacomini(1), Alexandre Doneda(1), \\ Rafael Ricardo Cantú(1), Alexandre Dessbesell(1), Roberto Carlos Lüdtke ${ }^{(1)}$ e Carlos Augusto Posser Silveira ${ }^{(2)}$ \\ (1)Universidade Federal de Santa Maria, Centro de Ciências Rurais, Departamento de Solos, Avenida Roraima, no 1.000, CEP 97105-900
Santa Maria, RS, Brasil. E-mail: agrogiacomini@gmail.com, celsoaita@gmail.com, pujolstefen@gmail.com, sjgiacomini@gmail.com,
alexandredoneda@gmail.com, rafaelcantu0@gmail.com, dessbeselale@gmail.com, betoludtke@gmail.com ${ }^{(2)}$ Embrapa Clima Temperado,
Rodovia BR 392, Km 78, Caixa Postal 403, CEP 96010-971 Pelotas, RS, Brasil. E-mail: augusto.posser@embrapa.br
}

Resumo - O objetivo deste trabalho foi avaliar a eficiência das zeólitas naturais clinoptilolita e estilbita em mitigar as perdas de amônia $\left(\mathrm{NH}_{3}\right)$ na fase inicial da compostagem de dejetos líquidos de suínos (DLS). Foram conduzidos três experimentos em escala piloto, com duração de 14 dias cada um. As zeólitas foram adicionadas aos DLS em doses crescentes, de 5 a $20 \%(\mathrm{~m} / \mathrm{v})$, sobre substrato constituído pela mistura de serragem $(70 \%)$ e maravalha (30\%). Foram realizadas três aplicações de DLS + zeólitas por experimento, seguidas de revolvimento. O substrato passou por outros três revolvimentos entre as aplicações. As zeólitas reduziram as emissões de $\mathrm{NH}_{3}$ e a sua eficiência foi diretamente relacionada à dose aplicada. A clinoptilolita apresentou maior eficiência do que a estilbita. Na média dos três experimentos, a clinoptilolita reduziu em 24 a $76 \%$ as emissões de $\mathrm{NH}_{3}$. Os resultados evidenciam o alto potencial de zeólitas naturais, principalmente da clinoptilolita, em mitigar a volatilização de $\mathrm{NH}_{3}$ durante a compostagem de DLS.

Termos para indexação: capacidade de troca catiônica, clinoptilolita, compostagem automatizada, estilbita, granulometria, perdas de nitrogênio.

\section{Mitigation of ammonia emissions by natural zeolites during pig slurry composting}

\begin{abstract}
The objective of this work was to evaluate the efficiency of the natural zeolites clinoptilolite and stilbite to mitigate ammonia $\left(\mathrm{NH}_{3}\right)$ losses during the initial phase of pig slurry (PS) composting. Three experiments were performed in pilot scale, each lasting 14 days. The zeolites were added to the PS in increasing rates, from 5 to $20 \%(\mathrm{w} / \mathrm{v})$, on a substrate consisting of a mixture of sawdust $(70 \%)$ and shavings $(30 \%)$. Three applications of PS + zeolites were performed per experiment, followed by a turning. The substrate went through three additional turnings, between applications. The zeolites reduced $\mathrm{NH}_{3}$ emissions and their efficiency was directly related to the applied rate. Clinoptilolite was more efficient than stilbite. In the average of the three experiments, clinoptilolite reduced in 24 to $76 \% \mathrm{NH}_{3}$ emissions. The results show the high potential of natural zeolites, mainly clinoptilolite, in mitigating $\mathrm{NH}_{3}$ volatilization during PS composting.

Index terms: cation exchange capacity, clinoptilolite, automatized composting, stilbite, granulometry, nitrogen losses.
\end{abstract}

\section{Introdução}

A suinocultura é uma importante atividade econômica, principalmente para o setor agropecuário da região Sul do Brasil, onde está concentrado o maior plantel nacional de suínos (Produção da pecuária municipal, 2010). O sistema de criação utilizado pela maioria dos produtores consiste no confinamento total dos animais, o que concentra, em pequenas áreas, grandes volumes de dejetos na forma líquida. Tradicionalmente, os dejetos líquidos de suínos (DLS) são armazenados em esterqueiras anaeróbicas, antes de serem aplicados ao solo como fonte de nutrientes às culturas, especialmente de $\mathrm{N}$. Aplicações sucessivas de DLS na mesma área levam a problemas ambientais, com destaque para as emissões gasosas de amônia $\left(\mathrm{NH}_{3}\right)$ e óxido nitroso $\left(\mathrm{N}_{2} \mathrm{O}\right)$ (Gonzatto et al., 2013; Aita et al., 2014), e para a possibilidade de contaminação do lençol freático por nitrato $\left(\mathrm{NO}_{3}^{-}\right)$(Zaman \& Blennerhassett, 2010). Portanto, o manejo adequado destes dejetos é imprescindível para a sustentabilidade da suinocultura brasileira.

A compostagem vem sendo preconizada como alternativa para reduzir o potencial poluente dos 
DLS. Nesse processo, os DLS são misturados a substratos com elevada relação $\mathrm{C} / \mathrm{N}$, como maravalha e serragem (Fukumoto et al., 2011). Recentemente, foi introduzido, no Brasil, o sistema de compostagem automatizada (Oliveira \& Higarashi, 2006), que consiste de aplicações frequentes de DLS nas leiras de compostagem, com simultâneo revolvimento destas. Esse sistema possibilita a aplicação de um maior volume de dejetos ao substrato e proporciona menor dispêndio com mão de obra, em comparação à compostagem tradicional.

A compostagem modifica as características químicas e físicas dos dejetos, e dá origem a um produto final com alto valor agronômico. No entanto, a prática está sujeita a perdas significativas de $\mathrm{N}$ por volatilização de $\mathrm{NH}_{3}$, o que reduz o potencial fertilizante do composto final (Fukumoto et al., 2011). Entre os fatores responsáveis pelas elevadas perdas de $\mathrm{NH}_{3}$, pode-se destacar a alta concentração de N amoniacal nos DLS (Jiang et al., 2013), a aeração das pilhas de compostagem durante os revolvimentos (Szanto et al., 2007), e a elevação do pH e da temperatura (Jiang et al., 2013) durante o processo. Encontrar alternativas para reduzir essas perdas é um aspecto ainda pouco pesquisado, tanto no Brasil quanto em outros países. Uma alternativa promissora consiste na adição de zeólitas naturais ao substrato. As zeólitas são encontradas em rochas sedimentares e formadas por tetraedros de $\mathrm{AlO}_{4} \mathrm{e}$ $\mathrm{SiO}_{4}$, cuja união dos anéis forma um sistema de canais e cavidades. Essa estrutura tridimensional, na forma de canais e de cavidades interconectados, proporciona estabilidade da estrutura cristalina, elevada capacidade de troca catiônica (CTC), capacidade de captura de gases e de vapores, propriedades catalíticas e alto grau de hidratação (Ming \& Mumpton, 1989). Essas características fazem com que as zeólitas retenham amônio nas cargas superficiais e o gás amônia em suas cavidades (Bernal et al., 1993; Bautista et al., 2011).

Existem várias espécies de zeólitas, mas a clinoptilolita e a estilbita destacam-se no cenário brasileiro. Antes de serem utilizadas, as zeólitas precisam ser submetidas à moagem, e esse processo interfere diretamente na CTC, que é dependente da granulometria do material (Malekian et al., 2011). A aplicação de zeólitas para reduzir a volatilização de $\mathrm{NH}_{3}$ durante a compostagem de dejetos de suínos, tanto sólidos (Bautista et al., 2011) como líquidos (Bernal et al., 1993), ainda está restrita à clinoptilolita, e não foram encontradas publicações com a estilbita. Além disso, nas publicações com clinoptilolita, avaliouse somente o processo clássico de compostagem, em que se realiza apenas uma adição de dejetos ao invés de adições frequentes, conforme preconiza a compostagem automatizada.

O objetivo deste trabalho foi avaliar a eficiência das zeólitas naturais clinoptilolita e estilbita em mitigar as perdas de $\mathrm{NH}_{3}$ na fase inicial da compostagem de dejetos líquidos de suínos (DLS).

\section{Material e Métodos}

Foram realizados três experimentos, com duração de 14 dias cada um, no Departamento de Solos da Universidade Federal de Santa Maria, RS. Os experimentos foram instalados no interior de uma edificação com pé-direito de $3 \mathrm{~m}$, coberta com telhas translúcidas e com muretas laterais de $1,5 \mathrm{~m}$ de altura. As aberturas presentes no espaço entre as muretas e a cobertura permitem a circulação de ar no interior da edificação. Os experimentos foram conduzidos em escala piloto, e a compostagem foi simulada em vasos de PVC, com diâmetro de $0,24 \mathrm{~m}$ e altura de $0,20 \mathrm{~m}$. $\mathrm{O}$ fundo dos vasos foi fechado.

O material orgânico utilizado como substrato continha maravalha de eucalipto $(30 \%)$ e serragem de diversas espécies de madeiras (70\%), ambos obtidos em madeireiras. Em cada vaso, foi adicionado $1 \mathrm{~kg}$ de substrato (em base úmida), constituído pela mistura de $0,3 \mathrm{~kg}$ de maravalha e $0,7 \mathrm{~kg}$ de serragem. A quantidade total de DLS adicionada por vaso, durante os 14 dias de cada experimento, foi de $3 \mathrm{~L}$, a qual foi dividida em três aplicações iguais de $1 \mathrm{~L}$ cada uma. A análise do substrato e dos DLS foi feita conforme metodologia descrita por Tedesco et al. (1995), e as suas principais características, bem como as quantidades de $\mathrm{N}$ adicionadas com os DLS em cada experimento, estão descritas na Tabela 1. Os DLS foram coletados em esterqueira anaeróbica, oriundos de animais em fase de terminação e constituídos pela mistura de fezes, urina e sobras de alimentação e de água dos bebedouros.

As duas espécies de zeólitas naturais utilizadas foram a clinoptilolita e a estilbita. A clinoptilolita foi fornecida pela Indústria Celta Brasil Ltda., localizada em Cotia, SP, e a estilbita foi proveniente de minério extraído da pedreira Ouro Preto, em Santa Cruz do Sul, RS. Na pedreira Ouro Preto, as zeólitas ocorrem em brechas de topo de derrame basáltico, capeado por um 
provável domo de natureza ácida (domo riolítico), que é a rocha lavrada para brita. A brecha é formada por clastos decimétricos de basalto vítreo, com cavidades do tipo amígdalas e vesículas preenchidas por zeólita heulandita, cimentados por crostas de $2-3 \mathrm{~cm}$ de espessura, correspondentes à zeólita.

As duas zeólitas foram adicionadas aos DLS nas proporções de 5, 10 e $20 \%$, em relação ao volume aplicado (m/v). Assim, em cada aplicação de DLS (1 L) ao substrato, eram adicionados 50,100 e $200 \mathrm{~g}$ de zeólita para as proporções de 5, 10 e $20 \%$, respectivamente. Essas dosagens correspondem à adição total de 150, 300 e 600 g de cada zeólita, para os 3 L de DLS aplicados em cada experimento. Além da aplicação inicial ao substrato da mistura de DLS + zeólitas, foram realizadas a segunda e a terceira aplicações, após cinco e nove dias, no experimento 1; quatro e dez dias, no experimento 2; e quatro e nove dias, no experimento 3. Após cada aplicação na superfície dos vasos, o substrato era uniformemente homogenizado com revolvimento manual, com o auxílio de sarrafo de madeira. Além do revolvimento pós-aplicação da mistura, foram realizados revolvimentos adicionais aos dois, sete e 12 dias, nos experimentos 1 e 3, e aos dois, sete e 11 dias, no experimento 2. Esses revolvimentos tinham o objetivo de facilitar a evaporação da fração líquida e oxigenar o sistema, o que simularia o processo de compostagem automatizada.

No experimento 1, foi avaliado o efeito do tipo e da dose de zeólitas sobre as perdas de $\mathrm{N}$ por volatilização. Foram comparados cinco tratamentos: T1, DLS apenas; T2, DLS + clinoptilolita fina (CLPf) a 5\% (DLS + CLPf 5\%); T3, DLS + estilbita fina (STIf) a $5 \%$ (DLS + STIf 5\%); T4, DLS + CLPf 10\%; e T6, DLS + STIf 10\%. As zeólitas clinoptilolita e estilbita finas apresentavam granulometrias de $\leq 0,0105$ e $\leq 0,3 \mathrm{~mm}$, respectivamente. $\mathrm{O}$ experimento 2 foi conduzido para consolidar os resultados obtidos no experimento 1, porém com ventilação em todas as câmaras utilizadas para avaliar as emissões de $\mathrm{NH}_{3}$, com os seguintes tratamentos: T1, DLS apenas; T2, DLS + CLPf 10\%; T3, DLS + STIf 10\%; T4, DLS + clinoptilolita grossa (CLPg) a 20\% (DLS + CLPg $20 \%$ ); e T5, DLS + STIf 20\%. A clinoptilolita grossa, usada no tratamento $\mathrm{T} 4$, apresenta granulometria entre 1 e $3 \mathrm{~mm}$. No experimento 3, foi comparada a eficiência das duas zeólitas, em reduzir a volatilização de $\mathrm{NH}_{3}$ na compostagem, por meio de três tratamentos, com três repetições: T1, DLS apenas; T2, DLS + CLPg 20\%; e T3, DLS + estilbita grossa (STIg) a 20\% (DLS + STIg 20\%). Utilizou-se o delineamento inteiramente casualizado nos três experimentos, com três repetições por tratamento.

As avaliações das perdas de $\mathrm{N}$ por volatilização de $\mathrm{NH}_{3}$ foram realizadas conforme adaptação da metodologia proposta por Nômmik (1973). Em cada avaliação, as câmaras de $\mathrm{PVC}$, com diâmetro de $0,240 \mathrm{~m}$ e altura de $0,205 \mathrm{~m}$, foram encaixadas sobre os vasos com os tratamentos avaliados. $\mathrm{Na}$ extremidade superior e em toda a parte externa de cada vaso, foi construída uma canaleta lateral com $0,03 \mathrm{~m}$ de largura e $0,02 \mathrm{~m}$ de altura, a qual permaneceu preenchida com

Tabela 1. Teores e quantidades de matéria seca, nitrogênio total e nitrogênio amoniacal de dejetos líquidos de suínos (DLS) adicionadas ao substrato.

\begin{tabular}{|c|c|c|c|c|c|c|c|}
\hline \multirow[t]{2}{*}{ Material } & \multicolumn{2}{|c|}{ Matéria seca } & \multicolumn{2}{|c|}{$\mathrm{N}$ total } & \multicolumn{2}{|c|}{$\mathrm{N}$ amoniacal } & \multirow[t]{2}{*}{$\mathrm{pH}$} \\
\hline & Teor $(\%)$ & Adição (g por vaso) & Teor $(\%)$ & Adição (g por vaso) & Teor $(\%)$ & Adição (g por vaso) & \\
\hline & \multicolumn{7}{|c|}{ Experimento 1} \\
\hline Substrato & 90,50 & 905,40 & 0,10 & 1,01 & 0,00 & 0,04 & 5,77 \\
\hline DLS, $1^{\text {a }}$ aplicação & 3,08 & 30,80 & 0,43 & 4,34 & 0,37 & 3,73 & 6,95 \\
\hline DLS, $2^{\text {a }}$ aplicação & 4,39 & 43,90 & 0,47 & 4,69 & 0,34 & 3,42 & 6,86 \\
\hline \multirow[t]{2}{*}{$\underline{\mathrm{DLS}}, 3^{\mathrm{a}}$ aplicação } & 4,08 & 40,80 & 0,45 & 4,50 & 0,29 & 2,86 & 6,87 \\
\hline & \multicolumn{7}{|c|}{ Experimento 2} \\
\hline Substrato & 89,54 & 895,4 & 0,10 & 1,03 & 0,02 & 0,22 & 5,76 \\
\hline DLS, $1^{\mathrm{a}}$ aplicação & 3,40 & 34,0 & 0,41 & 4,06 & 0,29 & 2,90 & 7,04 \\
\hline DLS, $2^{\mathrm{a}}$ aplicação & 3,49 & 34,9 & 0,43 & 4,31 & 0,29 & 2,89 & 7,07 \\
\hline \multirow[t]{2}{*}{ DLS, $3^{\mathrm{a}}$ aplicação } & 3,97 & 39,7 & 0,44 & 4,41 & 0,28 & 2,77 & 7,03 \\
\hline & \multicolumn{7}{|c|}{ Experimento 3} \\
\hline Substrato & 87,45 & 874,5 & 0,10 & 1,01 & 0,01 & 0,15 & 5,74 \\
\hline DLS, $1^{\mathrm{a}}$ aplicação & 4,45 & 44,5 & 0,47 & 4,75 & 0,28 & 2,78 & 7,09 \\
\hline DLS, $2^{\mathrm{a}}$ aplicação & 5,31 & 53,1 & 0,49 & 4,90 & 0,32 & 3,19 & 7,27 \\
\hline DLS, $3^{\mathrm{a}}$ aplicação & 5,23 & 52,3 & 0,51 & 5,09 & 0,33 & 3,32 & 7,13 \\
\hline
\end{tabular}


água durante o período de avaliação. A canaleta foi usada para garantir a completa vedação do conjunto vaso/câmara e evitar fugas de $\mathrm{NH}_{3}$. A uma altura de 0,05 $\mathrm{m}$ a partir da extremidade superior de cada câmara, uma tampa, também de PVC, foi colocada para impedir a entrada de raios solares; sem, no entanto, impedir o fluxo de ar no sistema.

No interior de cada câmara, havia duas esponjas, de densidade 28 e espessura de $0,02 \mathrm{~m}$, com as mesmas dimensões e formato das câmaras. A esponja inferior, disposta à altura de $0,10 \mathrm{~m}$ da superfície do composto, captava $\mathrm{o}_{3}$ emitido da compostagem, enquanto a esponja superior, disposta à altura de $0,12 \mathrm{~m}$ acima da esponja inferior, foi utilizada para impedir a entrada externa de $\mathrm{NH}_{3}$. As esponjas superior e inferior encontravam-se embebidas em $140 \mathrm{~mL}$ de uma solução com ácido fosfórico e glicerina (50 e $40 \mathrm{~mL} \mathrm{~L}^{-1}$, respectivamente). As esponjas inferiores foram coletadas e substituídas periodicamente (dez coletas nos experimentos 1 e 2 , e 11 coletas no experimento 3), e levadas ao laboratório para lavagem sequencial, por cinco vezes, com $\mathrm{KCl} 1 \mathrm{~mol} \mathrm{~L}^{-1}$, até completar o volume de $1.000 \mathrm{~mL}$. Desse volume, foi retirada a alíquota de $20 \mathrm{~mL}$, a qual foi destilada em destilador de arraste de vapor semimicro Kjeldahl, após a adição de óxido de magnésio $(\mathrm{MgO})$, conforme descrito em Tedesco et al. (1995).

As câmaras utilizadas para avaliar a emissão de $\mathrm{NH}_{3}$ eram estáticas, ou seja, não apresentavam circulação de ar na sua atmosfera interna. Alguns resultados de pesquisa sugerem que a falta de circulação de ar no interior das câmaras subestima as reais emissões de $\mathrm{NH}_{3}$ (Smith et al., 2007). Por isso, paralelamente ao experimento 1, foi realizado um estudo para avaliar especificamente o efeito da ventilação interna nas câmaras sobre as quantidades captadas de $\mathrm{NH}_{3}$. Para tal, foram comparados, com três repetições, dois tratamentos simulando a compostagem e conduzidos sob condições idênticas ao experimento 1. Em um dos tratamentos, as câmaras eram idênticas às utilizadas no experimento 1, enquanto, no outro tratamento, foi fixado um ventilador tipo cooler no interior das câmaras, à altura de aproximadamente $0,10 \mathrm{~m}$ a partir do limite superior da massa de compostagem. Os coolers eram alimentados por corrente elétrica de 12 volts e permaneceram acionados durante todo o período experimental (14 dias).

Durante os 14 dias de avaliação, em cada um dos três experimentos, os períodos em que os vasos permaneceram abertos e sem as câmaras foi de, no máximo, $10 \mathrm{~min}$, durante as adições da mistura de DLS e zeólitas, seguidas de revolvimento, e durante cada um dos três revolvimentos adicionais realizados. Assumiu-se que as eventuais perdas de $\mathrm{NH}_{3}$ que possam ter ocorrido nesse curto espaço de tempo tenham sido mínimas, sem impactos significativos na comparação entre os tratamentos.

Os resultados foram submetidos à análise de variância, e as médias foram comparadas pelo teste de LSD, a 5\% de probabilidade, com uso do programa Sigma Plot, versão 11.0 (Jandel Scientific, San Rafael, CA, EUA).

\section{Resultados e Discussão}

A volatilização de $\mathrm{NH}_{3}$ constituiu uma via importante de perda de $\mathrm{N}$ durante a compostagem de dejetos de suínos, no experimento 1 (Figura 1). Esse resultado está de acordo com outros estudos (Szanto et al., 2007; Fukumoto et al., 2011; Jiang et al., 2013). As perdas são atribuídas a diversos aspectos ligados aos dejetos, com destaque para: o $\mathrm{pH}$, normalmente elevado (Jiang et al., 2013); os altos teores de $\mathrm{N}$ amoniacal (Bernal et al., 1993); e a elevada temperatura da massa de compostagem (Szanto et al., 2007). No presente trabalho, o $\mathrm{pH}$ dos dejetos aplicados no experimento 1 foi próximo a 7,0, e a proporção de $\mathrm{N}$ amoniacal em relação ao $\mathrm{N}$ total foi, em média, de 74\% (Tabela 1). Os revolvimentos frequentes na massa de compostagem fizeram com que a temperatura desta não diferisse da temperatura ambiente.

Quanto à cinética de emissão de $\mathrm{NH}_{3}$, observouse que os fluxos nessa forma gasosa aumentaram em todos os tratamentos, nas avaliações feitas após cada aplicação dos dejetos, com exceção da primeira avaliação, realizada 23,5 horas após a primeira aplicação de DLS, em que os fluxos foram baixos e não diferiram entre os tratamentos (Figura 1). Apesar de essa avaliação ter coincidido com valores de temperatura mais elevados durante o experimento, ela também coincidiu com os menores valores de $\mathrm{pH}$, próximos a 7,0, que não favorecem a presença de $\mathrm{N}$ amoniacal na forma de $\mathrm{NH}_{3}$, em soluções aquosas (Liu et al., 2007). A impregnação inicial do substrato com a fração líquida dos dejetos, onde está concentrado o $\mathrm{N}$ amoniacal, também pode ter contribuído para as baixas emissões iniciais de $\mathrm{NH}_{3}$. Com o aumento do $\mathrm{pH}$ da massa de compostagem para valores próximos a 8,7, da 
primeira para a segunda avaliação, registrou-se o maior pico nos fluxos de $\mathrm{N}$ amoniacal do experimento, o qual aumentou aproximadamente 18 vezes no tratamento testemunha, em que os dejetos não receberam a adição de zeólitas. Li et al. (2008) relataram que as emissões de $\mathrm{NH}_{3}$ somente aumentaram 48 horas após a adição dos dejetos de vacas em lactação à massa de compostagem.

A zeólita clinoptilolita misturada aos dejetos reduziu os fluxos de $\mathrm{N}$ amoniacal para a atmosfera, com as maiores reduções observadas nas maiores doses (Figura 1). A maior eficiência ocorreu com
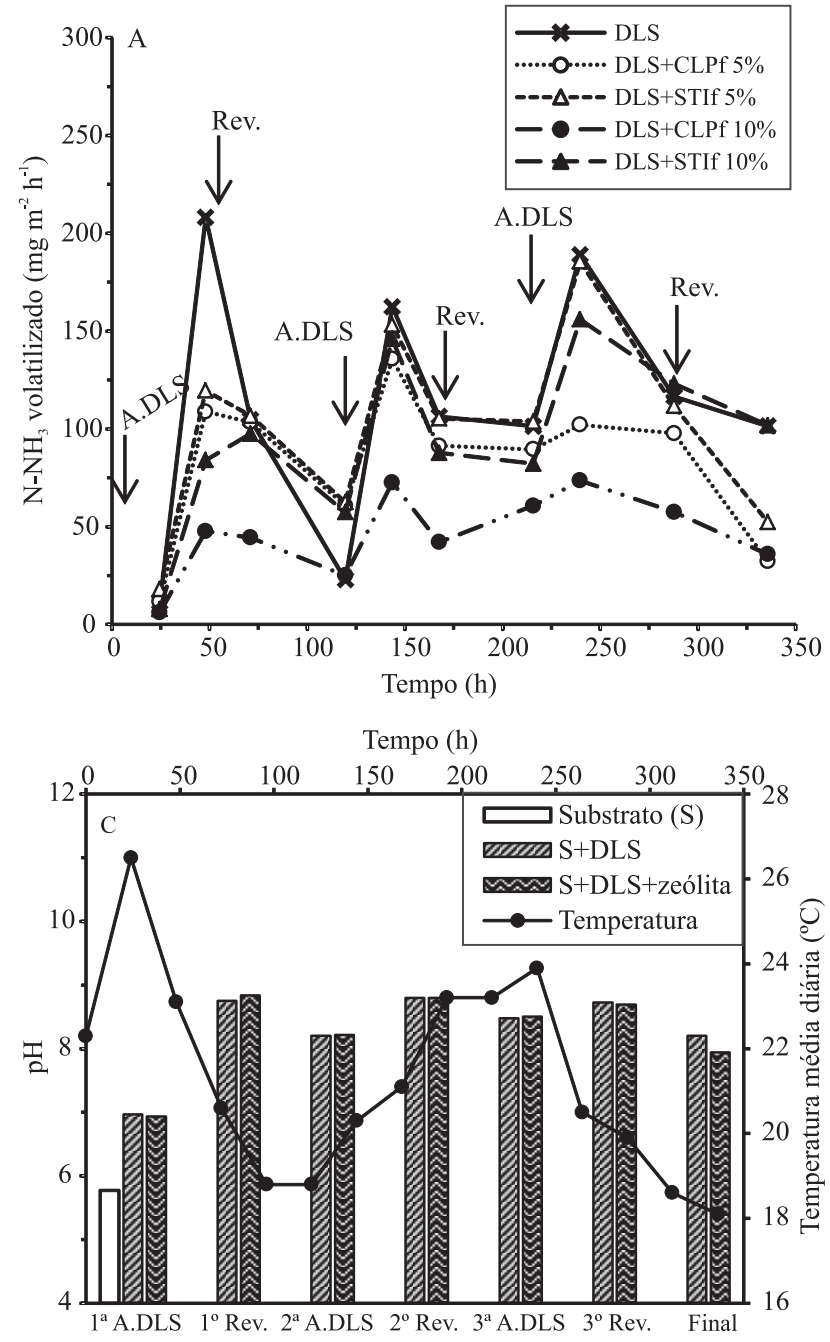

Sequência de atividades a dose de $10 \%$ (CLPf 10\%), em que a redução na emissão de $\mathrm{N}$ amoniacal na segunda avaliação foi de $77,1 \%$, em comparação à testemunha. Essa avaliação foi realizada 48 horas após a primeira adição dos DLS. Após a segunda aplicação, a redução na emissão foi de $55,3 \%$ e, após a terceira, de $61,2 \%$. Quando utilizada na dose de 5\%, a eficiência dessa zeólita foi menor, com reduções, nessas três mesmas avaliações, de 47,7, 16,4 e $46 \%$, respectivamente. A redução da volatilização de N amoniacal pela clinoptilolita, durante a compostagem, já foi relatada em outros estudos com dejetos suínos
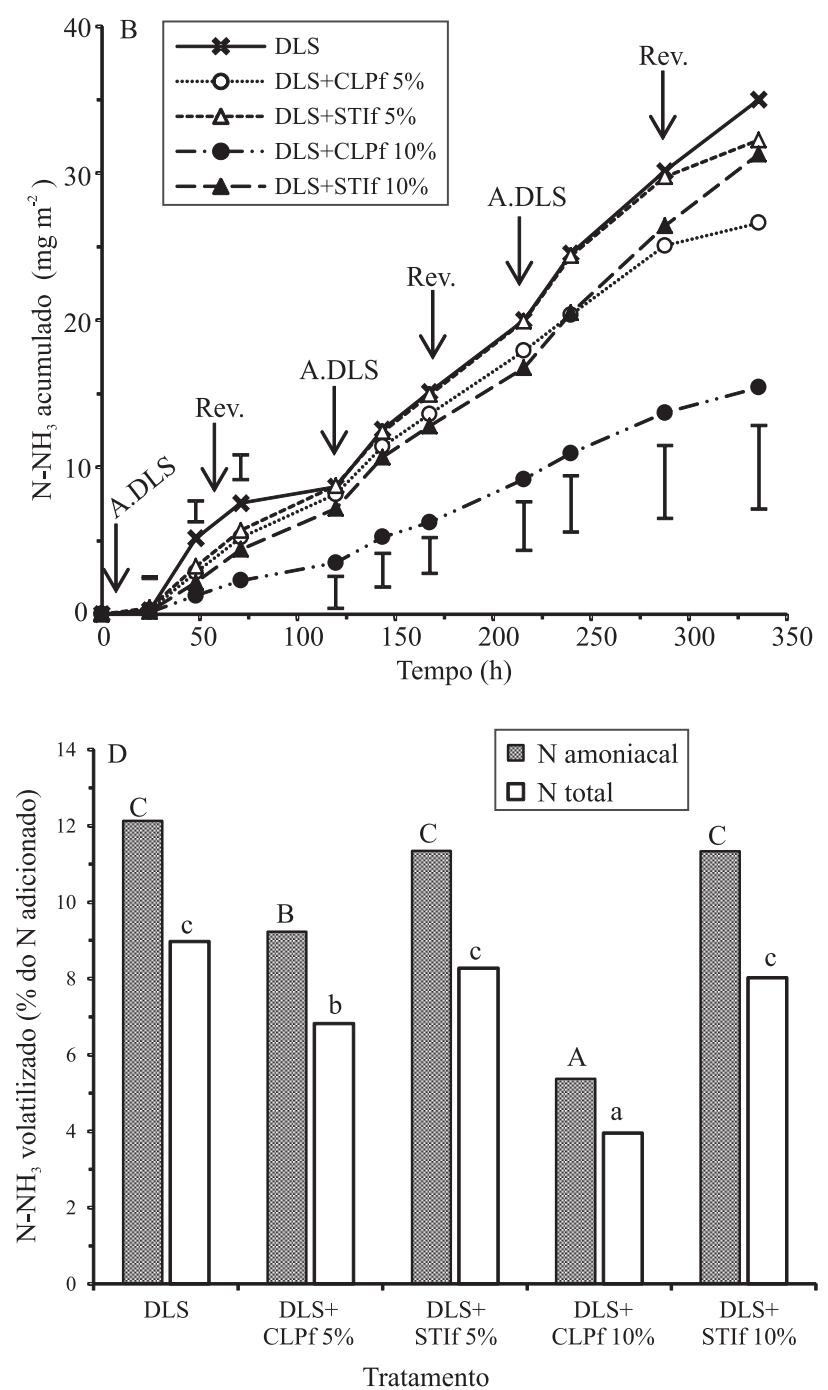

Figura 1. Fluxos de $\mathrm{N}-\mathrm{NH}_{3}(\mathrm{~A})$, emissão acumulada de $\mathrm{N}-\mathrm{NH}_{3}$ (B), temperatura média diária no ambiente e pH no composto (C), e fator de emissão de $\mathrm{N}-\mathrm{NH}_{3}$ (D) durante a compostagem dos dejetos líquidos de suínos (DLS), com e sem adição das zeólitas clinoptilolita (CLP) e estilbita (STI) finas (f), nas doses de 5 e 10\%, no experimento 1. Barras verticais ou letras representam a diferença mínima significativa pelo teste LSD, a $5 \%$ de probabilidade, para o fator de emissão de $\mathrm{N}_{-} \mathrm{NH}_{3}$ relativo ao $\mathrm{N}$ total e ao $\mathrm{N}$ amoniacal adicionados com os DLS, respectivamente. Flechas indicam os momentos de aplicação (A.) dos DLS e de revolvimento (Rev.) da massa de compostagem. 
(Bernal et al., 1993; Bautista et al., 2011) e, também, com diferentes materiais orgânicos (Kithome et al., 1998). Essa redução é atribuída à capacidade desses aluminossilicatos de adsorver cátions, absorver líquidos e capturar gases na sua estrutura tridimensional, na forma de canais e de cavidades interconectados (Ming \& Mumpton, 1989).

$\mathrm{O}$ efeito das zeólitas no controle das perdas de $\mathrm{N}$ por volatilização de $\mathrm{NH}_{3}$ pode ser mais bem visualizado na Figura $1 \mathrm{~B}$, em que é apresentada a evolução das perdas cumulativas de $\mathrm{N}$ amoniacal durante o experimento. Ao final do experimento, após 335,6 horas da primeira adição de DLS, a redução das emissões proporcionada pela maior dose de clinoptilolita (10\%) foi de $19,6 \mathrm{~g} \mathrm{~m}^{-2}$ de $\mathrm{N}(56 \%)$, o que corresponde a $6,82 \%$ da quantidade de $\mathrm{N}$ amoniacal aplicada com os DLS, durante os 14 dias de compostagem. Quanto aos demais tratamentos, apenas o com a aplicação de clinoptilolita na dose de 5\% diferiu significativamente do tratamento testemunha, com redução acumulada de $8,4 \mathrm{~g} \mathrm{~m}^{-2}$ de $\mathrm{N}(24 \%)$.

Embora também seja um aluminossilicato, a estilbita não foi eficiente em mitigar as perdas de $\mathrm{N}$ amoniacal na fase inicial da compostagem, no experimento 1 (Figura 1). Após 335,6 horas da primeira aplicação de DLS, a redução das emissões proporcionada pela maior dose de estilbita (10\%), em comparação à testemunha, foi de $3,7 \mathrm{~g} \mathrm{~m}^{-2}$ de N (11\%), o que corresponde a $1,29 \%$ da quantidade de $\mathrm{N}$ amoniacal aplicada com os DLS, nos 14 dias de compostagem. Essa baixa eficiência, em comparação à clinoptilolita, pode ser explicada pela menor afinidade da estilbita por cátions, como o $\mathrm{NH}_{4}^{+}$, e pelo fato de ela não ser uma zeólita totalmente pura - a utilizada no presente trabalho também apresentava outros elementos nutrientes presentes na matriz do basalto.

Bautista et al. (2011) constataram que a clinoptilolita é capaz de reduzir a volatilização de amônia durante a compostagem de esterco sólido de suínos $(81 \%$ de umidade), após a aplicação de $5 \%(\mathrm{~m} / \mathrm{m})$ da zeólita, unicamente no primeiro dia da compostagem. Contudo, como no presente trabalho, os autores não utilizaram a clinoptilolita pura, mas sim a misturada a sulfato de alumínio, na proporção de $2,5 \%(\mathrm{~m} / \mathrm{m})$. Ao final de 18 dias de compostagem, a mistura foi altamente eficiente na mitigação das perdas de $\mathrm{N}$ por volatilização de $\mathrm{NH}_{3}$, com redução de $92,4 \%$. Esses resultados, juntamente com os obtidos no presente trabalho, evidenciam o elevado potencial da clinoptilolita em preservar o $\mathrm{N}$ de dejetos animais, durante a compostagem, e este efeito está relacionado à dose empregada da zeólita, o que está de acordo com Bernal et al. (1993). Quanto à estilbita, não foi possível comparar o seu efeito ao relatado em outros trabalhos, já que estes não foram encontrados para compostagem.

A perda acumulada de $\mathrm{N}$ amoniacal (Figura 1) no tratamento testemunha correspondeu a $12,1 \%$ do $\mathrm{N}$ amoniacal, e a $9 \%$ do $\mathrm{N}$ total aplicado com os dejetos (Figura 1 D). Esse valor foi próximo ao encontrado por Fukumoto et al. (2009), de 9,57\% do $\mathrm{N}$ total, durante os primeiros 18 dias de compostagem. Jiang et al. (2013) observaram perdas maiores, entre 20 e $39 \%$ do $\mathrm{N}$ total aplicado, enquanto Szanto et al. (2007) obtiveram perdas de apenas 3,9\% do $\mathrm{N}$ total adicionado inicialmente a dejetos de suínos. Essas diferenças podem ser atribuídas às características dos dejetos utilizados nos diferentes trabalhos, ao ambiente predominante em cada um deles e aos métodos empregados na avaliação da $\mathrm{NH}_{3}$ volatilizada.

As câmaras utilizadas para avaliar a $\mathrm{NH}_{3}$ emitida durante a compostagem, no experimento 1, não apresentavam sistema de circulação de ar internamente, o que, conforme Smith et al. (2007), dificulta a captura de $\mathrm{NH}_{3}$ pelas esponjas contendo a mistura de $\mathrm{H}_{3} \mathrm{PO}_{4}+$ glicerina e subestima as perdas reais de N. Portanto, foi realizado um estudo adicional, em que a compostagem foi conduzida em condições similares àquelas do experimento 1, porém com apenas dois tratamentos: um com e outro sem ventilador do tipo cooler no interior das câmaras. Verificou-se que a falta de circulação de ar no interior das câmaras resultou em menores fluxos de $\mathrm{NH}_{3}$, em todas as avaliações realizadas. $\mathrm{O}$ coeficiente angular da relação entre os valores de emissão de $\mathrm{NH}_{3}$, determinados com e sem cooler, evidenciou que os valores obtidos sem ventilação devem ser corrigidos por um fator igual a $2,78(\mathrm{y}=2,7858 \mathrm{x}-0,2458)$. Assim, no experimento 1 , a emissão acumulada de $\mathrm{N}$ amoniacal aumentaria para $97 \mathrm{~g} \mathrm{~m}^{-2} \mathrm{de} \mathrm{N}$, no tratamento testemunha, e para $43 \mathrm{~g} \mathrm{~m}^{-2}$ de $\mathrm{N}$, no tratamento com $10 \%$ de clinoptilolita (Figura $1 \mathrm{~B}$ ). Esses resultados deixam claro que o uso de câmaras semiestáticas, para a determinação das perdas de $\mathrm{N}$ por volatilização de $\mathrm{NH}_{3}$, em compostagem, depende de um dispositivo que possibilite a circulação do ar no interior da câmara, no espaço existente entre o limite superior das pilhas de compostagem e a esponja coletora de $\mathrm{NH}_{3}$.

Durante as 336 horas do experimento 2, os fluxos de $\mathrm{N}-\mathrm{NH}_{3}$ e as quantidades acumuladas de $\mathrm{NH}_{3}$ volatilizadas também foram maiores no tratamento 
testemunha, tendo superado significativamente as obtidas nos tratamentos com aplicação de clinoptilolita. Entretanto, diferentemente do observado no experimento 1 , essas quantidades também foram superiores às dos tratamentos com estilbita. Ao final do experimento, a maior emissão acumulada de $\mathrm{N}$ amoniacal $\left(86,8 \mathrm{~g} \mathrm{~m}^{-2}\right.$ de $\left.\mathrm{N}\right)$, observada no tratamento testemunha, correspondeu a $35 \%$ do $\mathrm{N}$ amoniacal aplicado e superou a média dos dois tratamentos com estilbita, em 61,3 $\mathrm{g} \mathrm{m}^{-2}$ de N (41,6\%) (Figura 2 D). A menor emissão de $\mathrm{N}$ amoniacal ocorreu no tratamento com a maior dose de clinoptilolita (20\%), a qual foi $65,6 \mathrm{~g} \mathrm{~m}^{-2}$ de $\mathrm{N}$ menor $(76 \%)$ do que no tratamento testemunha. Embora esse resultado não possa ser atribuído apenas ao aumento para a dose de $20 \%$, uma vez que sua granulometria também era maior ( $1 \mathrm{a} 3 \mathrm{~mm}$ ), possivelmente a dose foi a principal responsável. As maiores emissões de $\mathrm{NH}_{3}$ detectadas no experimento 2 se deveram ao uso da ventilação no interior das câmaras, o que facilitou sua captura pelo $\mathrm{H}_{3} \mathrm{PO}_{4}$ contido nas esponjas.

Quando utilizadas na mesma granulometria (fina) e em doses equivalentes (10\%), a clinoptilolita foi mais eficiente do que a estilbita em reduzir as emissões
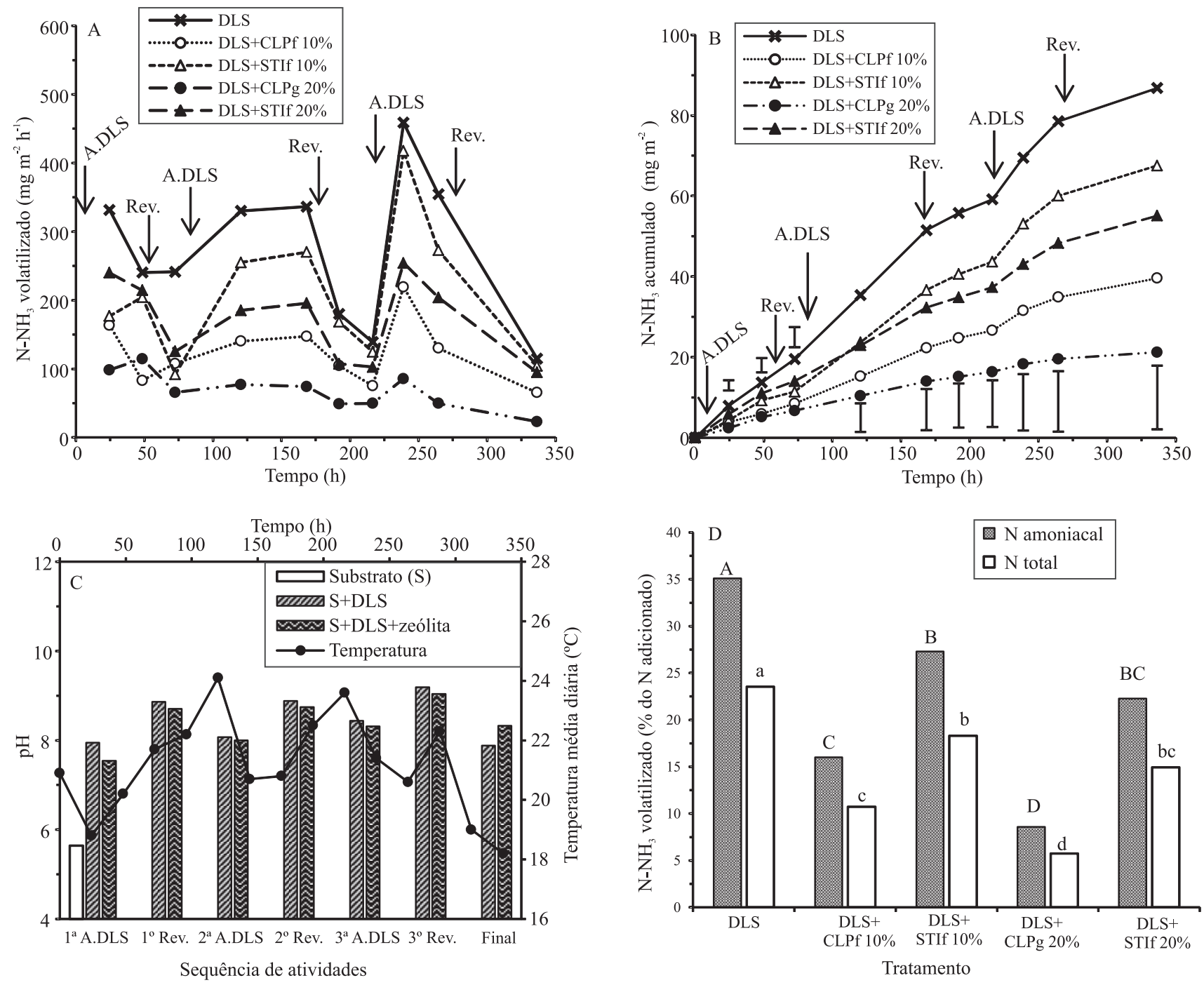

Figura 2. Fluxos de $\mathrm{N}$ amoniacal (A), emissão acumulada de $\mathrm{NH}_{3}(\mathrm{~B})$, temperatura média diária no ambiente e pH no composto (C), e fator de emissão de $\mathrm{NH}_{3}$ (D) durante a compostagem de dejetos líquidos de suínos (DLS), com e sem adição das zeólitas clinoptilolita (CLP) e estilbita (STI) finas (f) e clinoptilolita grossa (g), nas doses de 10 e $20 \%$, no experimento 2. Barras verticais ou letras representam a diferença mínima significativa pelo teste LSD, a 5\% de probabilidade. Flechas indicam os momentos de aplicação (A.) dos DLS e do revolvimento (Rev.) da massa de compostagem. 
de $\mathrm{NH}_{3}$, o que está de acordo com os resultados do experimento 1. Após 336 horas, a quantidade de $\mathrm{N}$ amoniacal que volatilizou no tratamento com estilbita superou a do tratamento com clinoptilolita, em 27,9 $\mathrm{g} \mathrm{m}^{-2}$ de $\mathrm{N}(70 \%)$. Mesmo dobrando a dose de estilbita (20\%), em comparação à de clinoptilolita a $10 \%$, a emissão com a primeira superou a com a segunda em 15,5 $\mathrm{g} \mathrm{m}^{-2}$ de $\mathrm{N}$ (39\%); porém, esta diferença não foi significativa (Figura $2 \mathrm{~B}$ ).

Em estudos futuros, seria interessante identificar as causas para essa maior afinidade/reatividade pelo $\mathrm{N}$ amoniacal de DLS da clinoptilolita. Outro aspecto importante a ser considerado é que a estilbita apresenta reservas importantes na região Sul do Brasil, enquanto a maior parte da clinoptilolita é importada, principalmente de Cuba. Dessa forma, as próximas pesquisas devem abordar aspectos econômicos da aplicação das duas zeólitas. Outro aspecto de interesse seria a avaliação das consequências do aumento na massa do composto - provocado pela adição das zeólitas - sobre a aplicabilidade deste como fertilizante.

A clinoptilolita foi mais eficiente do que a estilbita, tanto na granulometria fina (Figuras 1 e 2) quanto na grossa (Figura 3), o que deve estar relacionado à maior
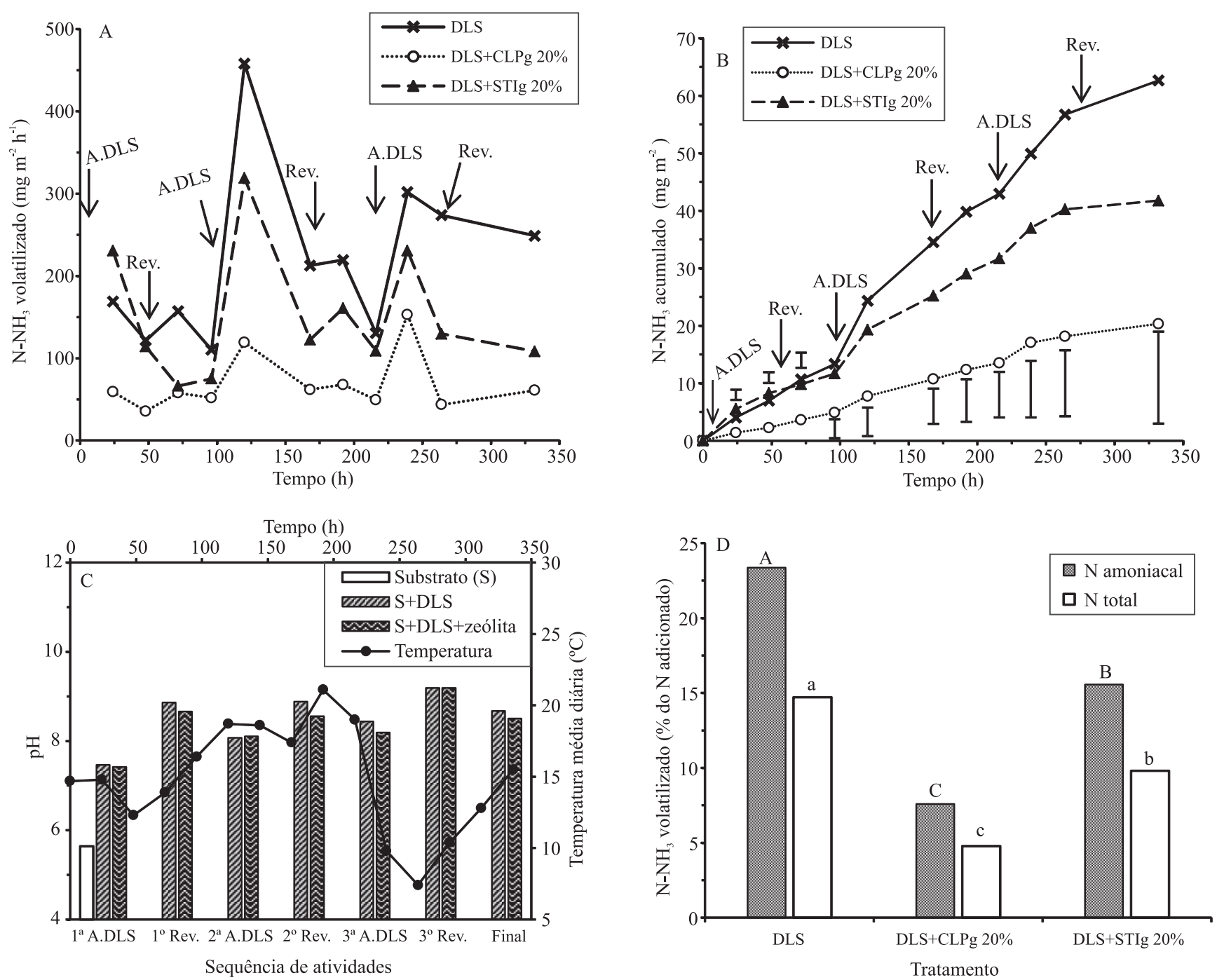

Figura 3. Fluxos de $\mathrm{N}$ amoniacal (A), emissão acumulada de $\mathrm{NH}_{3}(\mathrm{~B})$, temperatura média diária no ambiente e pH no composto (C), e fator de emissão de $\mathrm{NH}_{3}$ (D) durante a compostagem de dejetos líquidos de suínos (DLS), com e sem adição das zeólitas clinoptilolita (CLP) e estilbita (STI) grossas (g), nas doses de 10 e 20\%, no experimento 2. Barras verticais ou letras representam a diferença mínima significativa pelo teste LSD, a 5\% de probabilidade. Flechas indicam os momentos de aplicação (A.) dos DLS e do revolvimento (Rev.) da massa de compostagem. 
CTC da clinoptilolita. Ao final do experimento 3, a emissão de $\mathrm{N}$ amoniacal atingiu $62,6 \mathrm{~g} \mathrm{~m}^{-2}$ de $\mathrm{N}$ no tratamento testemunha, $41,7 \mathrm{~g} \mathrm{~m}^{-2}$ de $\mathrm{N}$ no tratamento com estilbita grossa e apenas $20,3 \mathrm{~g} \mathrm{~m}^{-2}$ de $\mathrm{N}$ no tratamento com a clinoptilolita grossa (Figura $3 \mathrm{~B}$ ). Além de maior reatividade, é possível que a clinoptilolita também apresente uma rede de canais e cavidades mais eficientes na retenção de $\mathrm{N}$ amoniacal do que a estilbita.

Finalmente, é importante salientar que o presente trabalho foi conduzido em escala piloto, em vasos de PVC, em que as variações de temperatura são diferentes de um processo de compostagem em escala real, conduzido em leiras, com adição periódica de dejetos e revolvimento simultâneo. Assim, a próxima etapa deste trabalho avaliará a eficiência das duas zeólitas, em plataforma de compostagem, e os seus efeitos sobre as emissões de gases de efeito estufa. Além disso, o potencial fertilizante dos compostos tratados com as zeólitas também deverá ser avaliado, pois, segundo Bautista et al. (2011), além de reduzir as emissões de $\mathrm{NH}_{3}$, as zeólitas conferem ao composto a característica de fontes de liberação lenta de N.

\section{Conclusões}

1. O uso de zeólitas naturais, com destaque para a clinoptilolita, é alternativa promissora para mitigar as perdas de $\mathrm{N}$ por volatilização de $\mathrm{NH}_{3}$ durante a compostagem de dejetos líquidos de suínos.

2. A eficiência das zeólitas em controlar a volatilização de $\mathrm{NH}_{3}$ durante a compostagem está diretamente relacionada à dose empregada.

\section{Agradecimentos}

À Coordenação de Aperfeiçoamento de Pessoal de Nível Superior (Capes), ao Conselho Nacional de Desenvolvimento Científico e Tecnológico (CNPq) e à Fundação de Amparo à Pesquisa do Estado do Rio Grande do Sul (Fapergs), pelo auxílio financeiro; à Empresa Celta Brasil Ltda, pelo fornecimento da zeólita clinoptilolita e à geóloga Magda Bergmann da Companhia de Pesquisa de Recursos Minerais (CPRM), pela indicação da zeolita estilbita.

\section{Referências}

AITA, C.; GONZATTO, R.; MIOLA, E.C.C.; SANTOS, D.B.; ROCHETTE, P.; ANGERS, D.A.; CHANTIGNY,
M.H.; PUJOL, S.B.; GIACOMINI, D.A.; GIACOMINI, S.J. Injection of dicyandiamide-treated pig slurry reduced ammonia volatilization without enhancing soil nitrous oxide emissions from no-till corn in Southern Brazil. Journal of Environmental Quality, v.43, p.789-800, 2014. DOI: 10.2134/ jeq2013.07.0301.

BAUTISTA, J.M.; KIM, H.; AHN, D.-H.; ZHANG, R.; OH, Y.-S. Changes in physicochemical properties and gaseous emissions of composting swine manure amended with alum and zeolite. Korean Journal of Chemical Engineering, v.28, p.189-194, 2011. DOI: 10.1007/s11814-010-0312-6.

BERNAL, M.P.; LOPEZ-REAL, J.M.; SCOTT, K.M. Application of natural zeolites for the reduction of ammonia emissions during the composting of organic wastes in a laboratory composting simulator. Bioresource Technology, v.43, p.35-39, 1993. DOI: 10.1016/0960-8524(93)90079-Q.

FUKUMOTO, Y.; INUBUSHI, K. Effect of nitrite accumulation on nitrous oxide emission and total nitrogen loss during swine manure composting. Soil Science and Plant Nutrition, v.55, p.428-434, 2009. DOI: 10.1111/j.1747-0765.2009.00376.x.

FUKUMOTO, Y.; SUZUKI, K.; KURODA, K.; WAKI, M; YASUDA, T. Effects of struvite formation and nitratation promotion on nitrogenous emissions such as $\mathrm{NH}_{3}, \mathrm{~N}_{2} \mathrm{O}$ and $\mathrm{NO}$ during swine manure composting. Bioresource Technology, v.102, p.1468-1474, 2011. DOI: 10.1016/j.biortech.2010.09.089.

GONZATTO, R.; MIOLA, E.C.C.; DONEDA, A.; PUJOL, S.B.; AITA, C.; GIACOMINI, S.J. Volatilização de amônia e emissão de óxido nitroso após aplicação de dejetos líquidos de suínos em solo cultivado com milho. Ciência Rural, v.43, p.1590-1596, 2013. DOI: $10.1590 / \mathrm{S} 0103-84782013000900009$.

JIANG, T.; SCHUCHARDT, F.; LI, G.X.; GUO, R.; LUO, Y.M. Gaseous emission during the composting of pig feces from Chinese Ganqinfen system. Chemosphere, v.90, p.1545-1551, 2013. DOI: 10.1016/j.chemosphere.2012.08.056.

KITHOME, M.; PAUL, J.W.; LAVKULICH, L.M.; BOMKE, A.A. Kinetics of ammonium adsorption and desorption by the natural zeolite clinoptilolite. Soil Science Society of America Journal, v.62, p.622-629, 1998. DOI: 10.2136/sssaj1998.0361 $5995006200030011 x$.

LI, X.; ZHANG, R.; PANG, Y. Characteristics of dairy manure composting with rice straw. Bioresource Technology, v.99, p.359-367, 2008. DOI: 10.1016/J.BIORTECH.2006.12.009.

LIU, Z.; WANG, L.; BEASLEY, D.; OVIEDO, E. Effect of moisture content on ammonia emissions from broiler litter: a laboratory study. Journal of Atmospheric Chemistry, v.58, p.41-53, 2007. DOI: 10.1007/S10874-007-9076-8.

MALEKIAN, R.; ABEDI-KOUPAI, J.; ESLAMIAN, S.S.; MOUSAVI, S.F.; ABBASPOUR, K.C.; AFYUNI, M. Ion-exchange process for ammonium removal and release using natural Iranian zeolite. Applied Clay Science, v.51, p.323-329, 2011. DOI: 10.1016/J.CLAY.2010.12.020.

MING, D.W.; MUMPTON, F.A. Zeolites in soils. In: DIXON, J.B.; WEED, S.B. (Ed.). Minerals in soil environments. $2^{\text {nd }}$ ed. Madison: Soil Science Society of America, 1989. p.873-911. 
NÔMMIK, H. The effect of pellet size on the ammonia loss from urea applied to forest soil. Plant and Soil, v.39, p.309-318, 1973. DOI: $10.1007 / \mathrm{BF} 00014798$.

OLIVEIRA, P.A.V. de; HIGARASHI, M.M. Unidade de compostagem para o tratamento dos dejetos de suínos. Concórdia: Embrapa Suínos e Aves, 2006. 39p. (Embrapa Suínos e Aves. Documentos, 114).

PRODUÇÃO DA PECUÁRIA MUNICIPAL. Rio de Janeiro: IBGE, v.38, 2010. 65p. Disponível em: <http://www.ibge.gov.br/ home/estatistica/economia/ppm/2010/ppm2010.pdf >. Acesso em: 10 out. 2012.

SMITH, E.; GORDON, R.; BOURQUE, C.; CAMPBELL, A. Comparison of three simple field methods for ammonia volatilization from manure. Canadian Journal of Soil Science, v.87, p.469-477, 2007. DOI: 10.4141/CJSS06038.
SZANTO, G.L.; HAMELERS, H.V.M.; RULKENS, W.H.; VEEKEN, A.H.M. $\mathrm{NH}_{3}, \mathrm{~N}_{2} \mathrm{O}$ and $\mathrm{CH}_{4}$ emissions during passively aerated composting of straw-rich pig manure. Bioresource Technology, v.98, p.2659-2670, 2007. DOI: 10.1016/J. BIORTECH.2006.09.021.

TEDESCO, M.J.; GIANELLO, C.; BISSANI, C.A.; BOHEN, H.; VOLKWEISS, S.J. Análises de solo, plantas e outros materiais. 2.ed. rev. ampl. Porto Alegre: Universidade Federal do Rio Grande do Sul, 1995. 174p.

ZAMAN, M.; BLENNERHASSETT, J.D. Effects of the different rates of urease and nitrification inhibitors on gaseous emissions of ammonia and nitrous oxide, nitrate leaching and pasture production from urine patches in an intensive grazed pasture system. Agriculture, Ecosystems and Environment, v.136, p.236-246, 2010. DOI: 10.1016/j.agee.2009.07.010.

Recebido em 8 de janeiro de 2014 e aprovado em 3 de julho de 2014 